'Departamento de

Administración y Economía,

Facultad de Ciencias Jurídicas y Empresariales, Universidad de La

Frontera. Temuco, Chile.

${ }^{2}$ Unidad de Economía de la

Salud, Centro de Investigación

y Gestión en Salud, Facultad

de Medicina, Universidad de La

Frontera. Temuco, Chile.

${ }^{3}$ Universidad Autónoma de

Madrid, Madrid, España.

aphd Economía de Empresa.

Recibido el 25 de noviembre de 2016, aceptado el 6 de octubre de 2017.

Financiamiento: Sin ayuda financiera, los autores no declaran conflicto de intereses.

Correspondencia a: Dr. Roberto Reveco S.

Universidad de La Frontera, Centro de Investigación y Gestión en Salud, Facultad de Medicina.

Montt 112, Temuco, Chile. Roberto.reveco@ufrontera.cl

\section{Impacto de diferentes métodos de asignación de costos indirectos estructurales de hospitales públicos, en el ranking costo-efectividad de 47 intervenciones en salud}

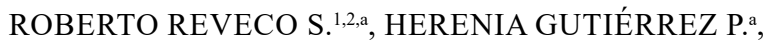 \\ JUAN PABLO RIEDEMANN G. ${ }^{2}$
}

Background: Costs allocation methods are important for economic evaluation of health care. Aim: To evaluate the impact of overhead costs rates of different hospitals on the cost-effectiveness rankings of health programs. Material and Methods: Using the cost reports from eight hospitals, a Montecarlo simulation was implemented, programming the complete micro-costing algorithm to calculate the final cost of 47 health care interventions, from the health sector perspective. The independent variables considered were the overhead cost rates per establishment and the actual overhead costs. Changing these variables, resulted in changes of the final cost of interventions and cost-effectiveness ratios. Finally the probabilities of changes in the cost-effectiveness ranking of each intervention were calculated. Results: Thirteen programs did not change their ranking order. However, 34 interventions modified their position with different occurrence probabilities. In the new proposed ranking, 21 programs changed their position from one to six places. Conclusions: Different overhead cost rates, representing different assignation forms, have a relative impact in the cost-effectiveness order. Montecarlo simulation can help to improve the accuracy of ranking assignment.

(Rev Med Chile 2017; 145: 1276-1288)

Key words: Cost Allocation; Costs and Cost Analysis; Cost-Benefit Analysis; Hospital Costs.

L as evaluaciones económicas de intervenciones de salud incluyen tanto los costos como los beneficios en salud ${ }^{1-9}$. En la mayoría de los estudios publicados, el costo del sector sanitario tiene un peso relativo importante.

El indicador que se determina para las evaluaciones mencionadas, es la llamada razón de costo-efectividad (RCE), que es el cociente entre los costos y los beneficios en salud expresados habitualmente en años de vida ajustados por calidad (AVAC) o años de vida ajustados por discapacidad $(\mathrm{AVAD})^{10-11}$.

En su forma clásica, se utiliza una RCE incremental, es decir, el incremento de costos dividido por el incremento en los beneficios, que se produce al comparar dos estrategias de salud. Luego, 
dicha razón se compara con un umbral, como el producto interno bruto per cápita u otro. Se adopta una nueva estrategia si la razón es menor que aquel umbral.

Cuando se trata de ejecutar un presupuesto limitado, uno de los instrumentos utilizados para apoyar la toma de decisiones, es una clasificación de las intervenciones de salud, ordenada en forma ascendente según el valor de sus RCE. Este ranking también es conocido como tabla de eficiencia o league tables ${ }^{12}$. Los decisores deberían entonces, asignar fondos a aquellas intervenciones con menores RCE (menor costo por unidad de beneficio en salud).

Los costos directos en el sector sanitario son el factor trabajo, utilización de equipamiento y diversos fármacos e insumos clínicos. Al costo directo anterior se debe agregar el costo indirecto estructural (CIE) que corresponde a los costos de energía, mantención, y todos los servicios de apoyo logístico y administrativo con que cuenta un hospital. Habitualmente estos costos se agregan mediante una tasa que corresponde al cociente entre los CIE totales y los costos directos totales.

La mayoría de los estudios publicados revelan los detalles de los costos directos, pero no necesariamente exponen los pasos acerca de la determinación, cálculo y asignación de CIE a los servicios de salud. Hay autores que señalan las dificultades existentes para encontrar información respecto a estos costos indirectos estructurales y también reconocen arbitrariedades en relación a las bases de reparto (cost drivers) utilizadas ${ }^{13}$. También, variados estudios los omiten parcial o totalmente. En la gran mayoría de estudios de costo efectividad marginal estos costos tienden a ser similares en ambos brazos de comparación, dado que la mayoría de ellos son relativamente fijos. Sin embargo, cuando se analizan diferentes intervenciones y se priorizan de acuerdo a sus RCE, es preferible incluir todos los costos.

La literatura revisada revela que muy pocos trabajos se han referido a establecer los efectos de las metodologías de asignación de CIE en otras variables dependientes, uno de ellos es el de Tan SS, van Ineveld BM, Redekop WK, et al. (2009), que se refiere al impacto de cuatro opciones de cálculo de tasas sobre cuatro servicios sanitarios ${ }^{14}$. El segundo trabajo $^{14}$, tuvo como objetivo comparar cuatro opciones de asignación o reparto hacia centros de costos para determinar cuál de ellas permitía una mejor predicción de costos por paciente ${ }^{15}$.

No se encontró ningún estudio que abordara el efecto de CIE sobre la clasificación de intervenciones según el valor de sus RCE.

Los estudios publicados muestran un amplio rango de porcentajes de agregación de costos indirectos estructurales a los servicios de salud, que fluctúa entre un mínimo de $10 \%$ y un máximo de $44 \%$ sobre los costos directos ${ }^{15-34}$.

La variabilidad en los ratios, puede tener su origen en la diversidad de establecimientos sanitarios (tamaño, nivel de infraestructura, consumo de energía, soporte de gestión), y métodos de reparto a nivel de centros de costos. Estos aspectos están poco estudiados, debido a la tendencia de analizar preferentemente costos directos, ya que ellos representan el porcentaje más importante dentro del costo total de los servicios de salud.

Este estudio se realizó para intentar responder las siguientes preguntas: (i) ¿importa realmente la magnitud de los CIE para construir un ranking de costo efectividad de intervenciones sanitarias?; (ii) ¿qué tanto se modifica el orden en el ranking, cuando se utilizan diferentes tasas de asignación de CIE en el costo de producción de las intervenciones estudiadas? (iii) ¿es necesario mayor investigación para estandarizar métodos de determinación de CIE de acuerdo a diferentes realidades hospitalarias?. Se estima que las respuestas a las interrogantes señaladas, contribuirán a mejorar la información sobre la cual, las autoridades toman decisiones de asignación de recursos.

\section{Material y Método}

Se consideraron los costos directos de 47 intervenciones en salud del Estudio de Costo Efectividad de Intervenciones en Salud, en sus etapas de diagnóstico, tratamiento y seguimiento (DTS), publicado por el Ministerio de Salud de Chile (MINSAL) en mayo de $2010^{35}$. Los costos en dicho estudio fueron obtenidos a partir de una muestra 22 hospitales públicos, tanto de la Región Metropolitana, como de la zona centro y sur del país. El estudio incluyó costos de 302 prestaciones que forman parte de las intervenciones en sus etapas de diagnóstico, tratamiento y seguimiento.

A partir de los reportes por centros de costos del sistema WinSig de una submuestra de 8 
hospitales, donde también fueron costeadas las prestaciones que forman parte de las intervenciones en salud, se extrajeron los costos indirectos estructurales de cada centro, y se construyeron las diferentes tasas de CIE, resultado del cociente entre los CIE de los centros de costos involucrados en las prestaciones que formaban parte de las intervenciones estudiadas, y sus respectivos costos directos.

Se diseñó y programó en Visual Basic de Excel 2010, una Simulación de Montecarlo, a través de la cual se implementó el algoritmo completo de costos de prestaciones de salud individuales, el costo de las intervenciones en salud, en cada etapa de diagnóstico, tratamiento y seguimiento, razones costo-efectividad y el lugar de clasificación en base a RCE. El ciclo detallado anteriormente se repitió 1.000 veces, cambiando en cada oportunidad la tasa de CIE (variable independiente). El proceso de Simulación se describe con detalle en la Figura 1, la cual se resume a través de los siguientes puntos:
1. La función de generación aleatoria de Montecarlo generará 1.000 tasas de CIE por cada prestación sanitaria.

2. Se modificará 1.000 veces el CIE de las prestaciones sanitarias que forman parte de las intervenciones en salud en las etapas de DTS.

3. Habrá 1.000 cambios en el costo de las etapas de diagnóstico, tratamiento y en el valor presente de los costos de seguimiento de cada intervención. Se modificará 1.000 veces el costo final de cada prestación de salud y de cada intervención (numerador de la RCE).

4. Cambiará 1.000 veces la razón costo-efectividad de cada Intervención y eventualmente, el lugar en la clasificación en base a la RCE.

5. Será generado un reporte con los 1.000 lugares que cada Intervención obtiene en el proceso anterior. El cual permitirá medir los impactos finales, en términos de la cantidad de lugares diferentes en el ranking de costo-efectividad que las intervenciones muestren y de sus respectivas probabilidades de ocurrir.

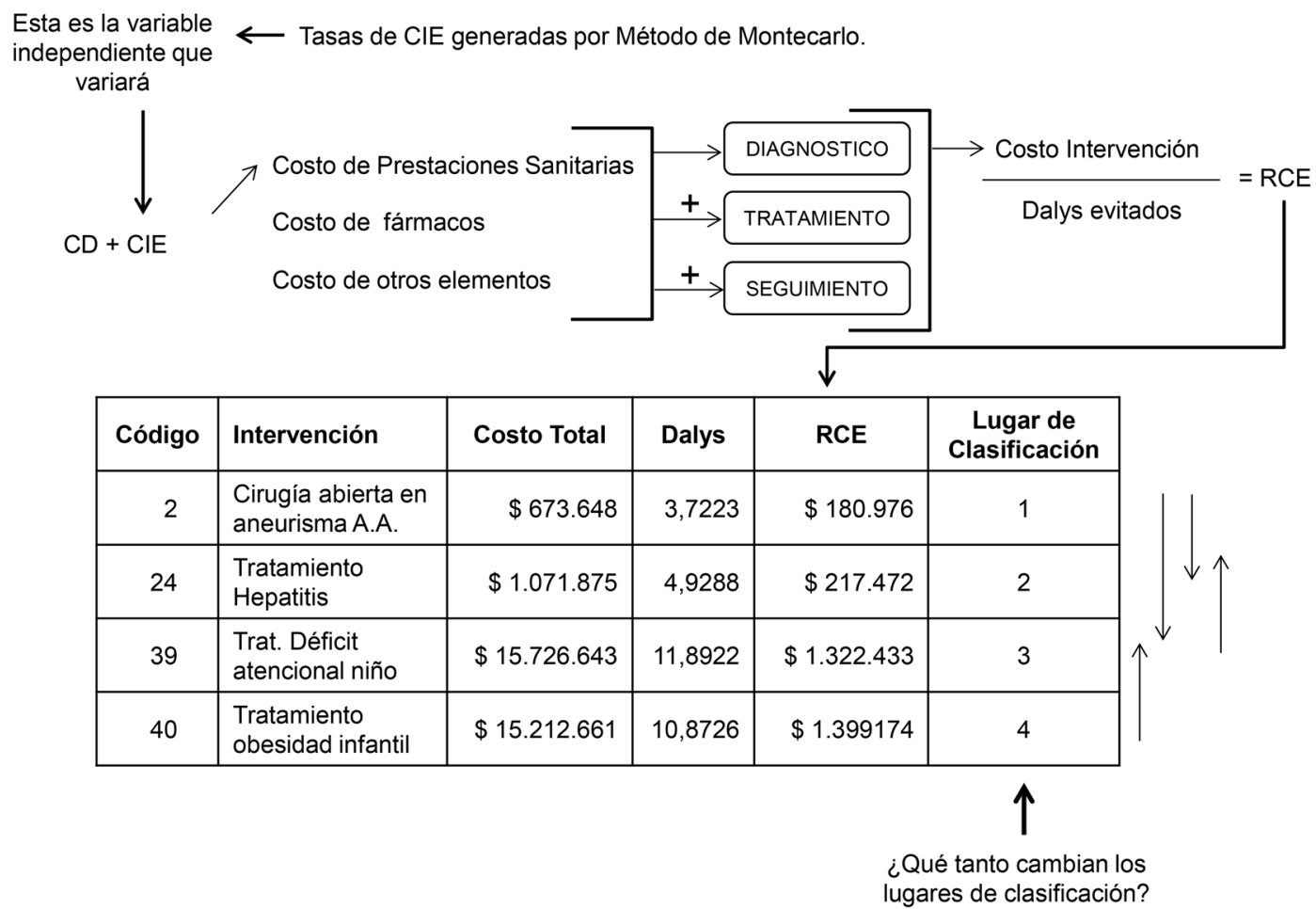

\begin{tabular}{|c|l|c|c|c|c|}
\hline Código & Intervención & Costo Total & Dalys & RCE & $\begin{array}{c}\text { Lugar de } \\
\text { Clasificación }\end{array}$ \\
\hline 2 & $\begin{array}{l}\text { Cirugia abierta en } \\
\text { aneurisma A.A. }\end{array}$ & $\$ 673.648$ & 3,7223 & $\$ 180.976$ & 1 \\
\hline 24 & $\begin{array}{l}\text { Tratamiento } \\
\text { Hepatitis }\end{array}$ & $\$ 1.071 .875$ & 4,9288 & $\$ 217.472$ & 2
\end{tabular}$\downarrow \uparrow$

Figura 1. Simulación de Montecarlo para impacto de CIE en ranking costo-efectividad de intervenciones en salud. 


\section{Resultados}

Se obtuvieron las tasas promedio, máximas y mínimas CIE, por cada uno de los centros donde se llevaron a cabo las 302 prestaciones sanitarias que forman parte de las intervenciones en salud estudiadas, información que se puede apreciar en la Tabla 1.

En la Tabla 2 se pueden visualizar los costos directos en pesos chilenos actualizados al $30 \mathrm{de}$ julio de 2016 de las 47 intervenciones en salud estudiadas, los DALYs evitados y el ranking de costo-efectividad obtenido en el Estudio del Minsal.

El reporte de lugares de clasificación según costo-efectividad que se describe a continuación, constituye el primer instrumento diseñado en este estudio para medir el impacto de las variaciones de tasas de CIE y, por ende, de métodos diferentes para su asignación. Este reporte se muestra a través de la Tabla 3.

Tabla 1. Tasas de costos indirectos estructurales

\begin{tabular}{|c|c|c|c|c|}
\hline Centro & Tasa promedio & Tasa Max. & Tasa Min. & DS \\
\hline Anatomía Patológica & 0,3816 & 0,5483 & 0,2040 & 0,0574 \\
\hline Banco de sangre & 0,1557 & 0,2057 & 0,1215 & 0,0140 \\
\hline CDT y consultas & 0,3364 & 0,7855 & 0,0600 & 0,1209 \\
\hline Cirugía & 0,4113 & 0,7855 & 0,0725 & 0,1188 \\
\hline Ginecología & 0,3569 & 0,4591 & 0,2760 & 0,0305 \\
\hline Hemodinamia & 0,2346 & 0,3919 & 0,1188 & 0,0455 \\
\hline Imagenología & 0,2620 & 0,4600 & 0,0704 & 0,0649 \\
\hline Laboratorios & 0,2140 & 0,3811 & 0,0726 & 0,0514 \\
\hline Medicina, C. Medicina y H. Med. & 0,3374 & 0,5193 & 0,2117 & 0,0513 \\
\hline Odontología & 0,3314 & 0,6433 & 0,2050 & 0,0731 \\
\hline Oftalmología & 0,3317 & 0,7826 & 0,0915 & 0,1152 \\
\hline Pabellones & 0,2809 & 0,5527 & 0,0754 & 0,0796 \\
\hline Salud Mental & 0,4026 & 0,7326 & 0,1938 & 0,0898 \\
\hline Traumatología & 0,4221 & 0,7793 & 0,2310 & 0,0914 \\
\hline
\end{tabular}

Razón entre CIE y costos directos de cada uno de los centros en que fueron costeadas 302 prestaciones de salud que formaron parte de las 47 intervenciones en salud estudiadas.

Tabla 2. Ranking de intervenciones conforme a sus razones de costo-efectividad estudio de Minsal $^{34}$ (\$ chilenos actualizados al 30 de julio de 2016)

\begin{tabular}{|c|c|c|c|c|}
\hline Código & Intervención & Costo & Dalys & RCE \\
\hline 42 & $\begin{array}{l}\text { Tratamiento de erradicación de la úlcera péptica (por } 14 \text { días, } \\
\text { cualquier esquema) }\end{array}$ & 59.686 & 0,6619 & 90.174 \\
\hline 3b & Cirugía laparoscópica en apendicitis aguda & 253.260 & 2,6176 & 96.753 \\
\hline 13 & $\begin{array}{l}\text { Tratamiento ortopédico de la displasia congénita de cadera } \\
\text { (según grado de severidad) }\end{array}$ & 123.010 & 1,1227 & 109.566 \\
\hline $3 a$ & Cirugía abierta en apendicitis aguda & 302.205 & 2,4961 & 121.071 \\
\hline 2 & $\begin{array}{l}\text { Tratamiento ortopédico y quirúrgico de las anomalías congénitas } \\
\text { tratables del aparato locomotor (pie bot) }\end{array}$ & 721.503 & 3,7223 & 193.832 \\
\hline 23 & Cirugía con uso de malla en hernias de la pared abdominal & 477.871 & 2,3221 & 205.793 \\
\hline 24 & Derivativa en hidrocefalia congénita al momento de diagnóstico & 1.191 .316 & 4,9288 & 241.705 \\
\hline 34 & Fototerapia en psoriasis & 293.714 & 0,5542 & 529.978 \\
\hline 20 & Terapia farmacológica tópica en glaucoma & 532.591 & 0,9296 & 572.925 \\
\hline
\end{tabular}




\begin{tabular}{|c|c|c|c|c|}
\hline Código & Intervención & Costo & Dalys & RCE \\
\hline $43 b$ & Escleroterapia en várices & 42.101 & 0,0707 & 595.492 \\
\hline 8 & Cirugía de cáncer de vejiga + terapia intravesical & 3.454 .036 & 2,7276 & 1.266 .328 \\
\hline 40 & Trasplante de hígado (insuficiencia hepática terminal) & 16.528 .571 & 10,8726 & 1.520 .204 \\
\hline 36 & Atención odontológica integral de la embarazada & 200.310 & 0,1252 & 1.599 .920 \\
\hline 6 & Estudio de ganglio centinela en cáncer de piel + tratamiento & 1.285 .956 & 0,7870 & 1.633 .998 \\
\hline $30 \mathrm{~b}$ & Tratamiento con banda gástrica en obesidad mórbida & 1.668 .600 & 1,0017 & 1.665 .768 \\
\hline 5 & Cirugía de cáncer de ovario + quimioterapia & 5.729 .995 & 3,1942 & 1.793 .875 \\
\hline $30 a$ & Tratamiento quirúrgico bypass en obesidad mórbida & 2.980 .524 & 1,6337 & 1.824 .401 \\
\hline 39 & Trasplante de corazón (insuficiencia cardiaca terminal) & 22.789 .228 & 11,8922 & 1.916 .317 \\
\hline 29 & Psicoterapia (individual y grupal) en el maltrato infantil & 266.286 & 0,1059 & 2.514 .507 \\
\hline 11 & Tratamiento de la depresión en adolescentes & 231.991 & 0,0900 & 2.577 .681 \\
\hline 28 & Tratamiento integral del lupus eritematoso sistémico & 2.497 .945 & 0,9368 & 2.666 .466 \\
\hline 44 & Consejería orientada a la mujer en violencia intrafamiliar (adultos) & 32.764 & 0,0118 & 2.776 .589 \\
\hline 41 & $\begin{array}{l}\text { Valvuloplastía con cirugía abierta de los trastornos valvulares } \\
\text { reumáticos y no reumáticos }\end{array}$ & 6.386 .130 & 2,2197 & 2.877 .024 \\
\hline 21 & Tratamiento de inmunoglobulina en Guillain-Barré & 10.415 .586 & 3,3889 & 3.073 .442 \\
\hline $43 a$ & Safenectomía en várices & 462.489 & 0,1454 & 3.180 .803 \\
\hline 18 & Tratamiento de la epilepsia en adultos & 2.041 .051 & 0,6002 & 3.400 .619 \\
\hline 12 & Tratamiento farmacológico y terapia de apoyo del desorden bipolar & 5.318 .150 & 1,4118 & 3.766 .929 \\
\hline 32 & $\begin{array}{l}\text { Cirugía en osteosarcoma mas quimioterapia pre y post cirugía } \\
\text { (en personas de } 15 \text { años y más) }\end{array}$ & 18.287 .734 & 4,6940 & 3.895 .981 \\
\hline 10 & Tratamiento integral para la cesación del tabaquismo & 277.703 & 0,0654 & 4.246 .216 \\
\hline 33 & Tratamiento con toxina botulínica tipo A en parálisis cerebral infantil & 5.726 .221 & 1,3321 & 4.298 .642 \\
\hline $26 a$ & Inducción ovárica en el tratamiento de la infertilidad & 1.444 .413 & 0,2793 & 5.171 .545 \\
\hline 14 & Tratamiento integral de la enfermedad de Alzheimer & 419.181 & 0,0781 & 5.367 .238 \\
\hline $1 b$ & Cirugía abierta en aneurisma aórtico abdominal & 2.402 .599 & 0,3833 & 6.268 .194 \\
\hline 9 & Cirugía de cáncer de vesícula biliar y vías biliares + quimioterapia & 5.861 .425 & 0,9029 & 6.491 .776 \\
\hline $26 b$ & Fertilización in vitro en el tratamiento de la infertilidad & 2.320 .399 & 0,3527 & 6.578 .959 \\
\hline 4 & Cirugía de cáncer de colon + quimioterapia & 13.388 .044 & 1,8667 & 7.172 .038 \\
\hline 22 & Tratamiento de la hepatitis c (peginf + ribavirina) & 13.795 .843 & 1,4768 & 9.341 .714 \\
\hline 17 & Angioplastia por stent en enfermedad isquémica del corazón & 3.549 .819 & 0,3195 & 11.110 .544 \\
\hline 35 & $\begin{array}{l}\text { Reemplazo hormonal en el climaterio (estrógenos conjugados más } \\
\text { progestágenos) }\end{array}$ & 669.573 & 0,0520 & 12.876 .394 \\
\hline 7 & Cirugía de cáncer de pulmón + quimioterapia & 9.816 .986 & 0,7065 & 13.895 .239 \\
\hline 37 & $\begin{array}{l}\text { Tratamiento farmacológico e intervención psicosocial del síndrome } \\
\text { de déficit atencional del niño }\end{array}$ & 1.738 .791 & 0,1096 & 15.864 .884 \\
\hline 16 & Tratamiento farmacológico de la enfermedad de Parkinson & 5.640 .163 & 0,2924 & 19.289.201 \\
\hline 1a & Stent endovascular en aneurisma aórtico abdominal & 10.010 .796 & 0,4459 & 22.450 .765 \\
\hline 19 & Interferón beta-1a para esclerosis múltiple rr & 21.422 .265 & 0,7104 & 30.155 .215 \\
\hline 38 & $\begin{array}{l}\text { Tratamiento del sobrepeso y obesidad infantil (por } 4 \text { meses, incluye } \\
\text { dieta, ejercicio y apoyo psicológico) }\end{array}$ & 242.591 & 0,0018 & 134.772 .917 \\
\hline 31 & $\begin{array}{l}\text { Terapia de reemplazo hormonal (calcio y vitamina D) en la } \\
\text { osteoporosis }\end{array}$ & 1.251 .256 & 0,0025 & 500.502 .500 \\
\hline 15 & Terapia de reemplazo enzimático en enfermedad de Gaucher & 185.235 .711 & 0,9348 & 4.477.145.605 \\
\hline
\end{tabular}




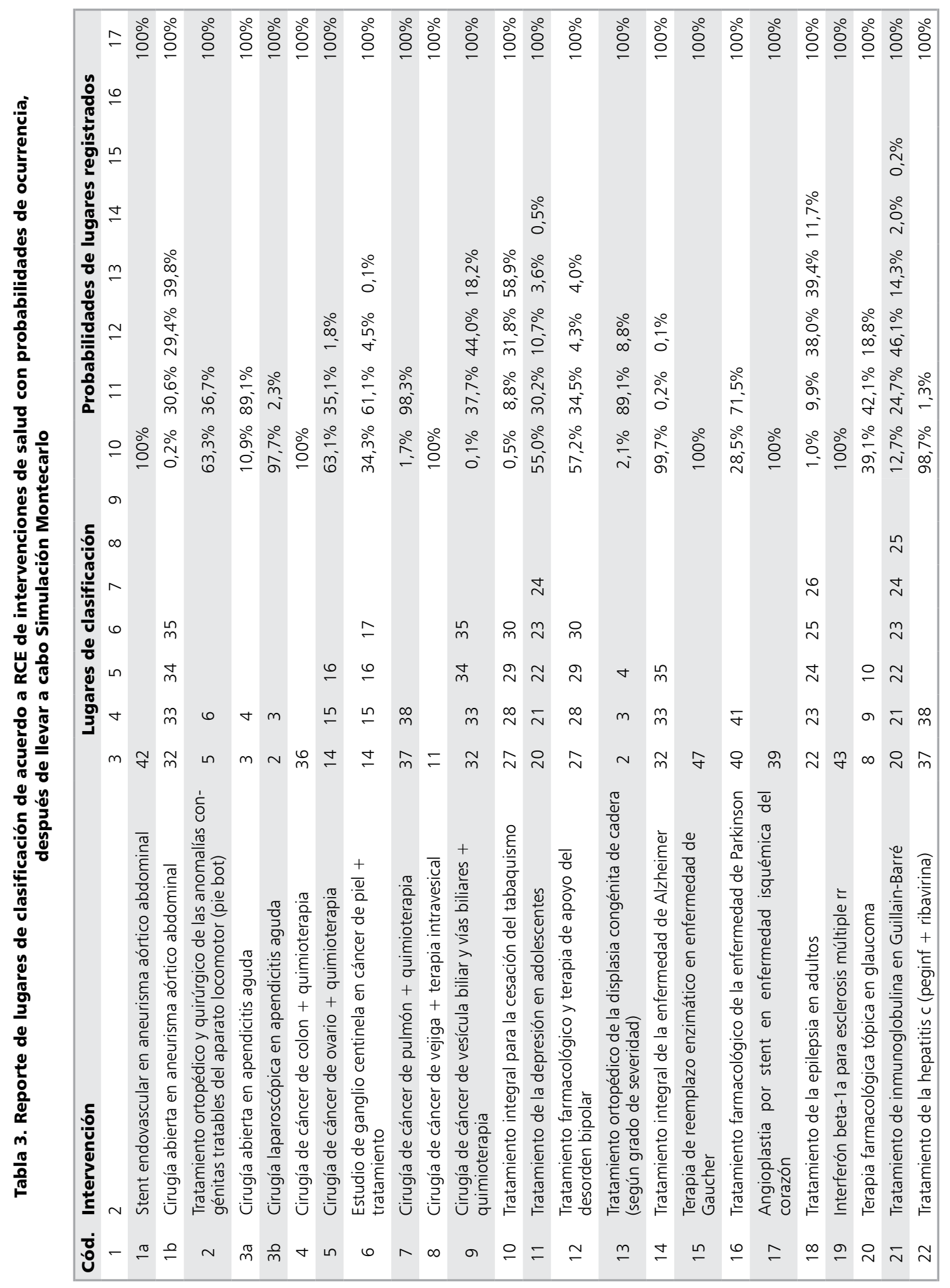




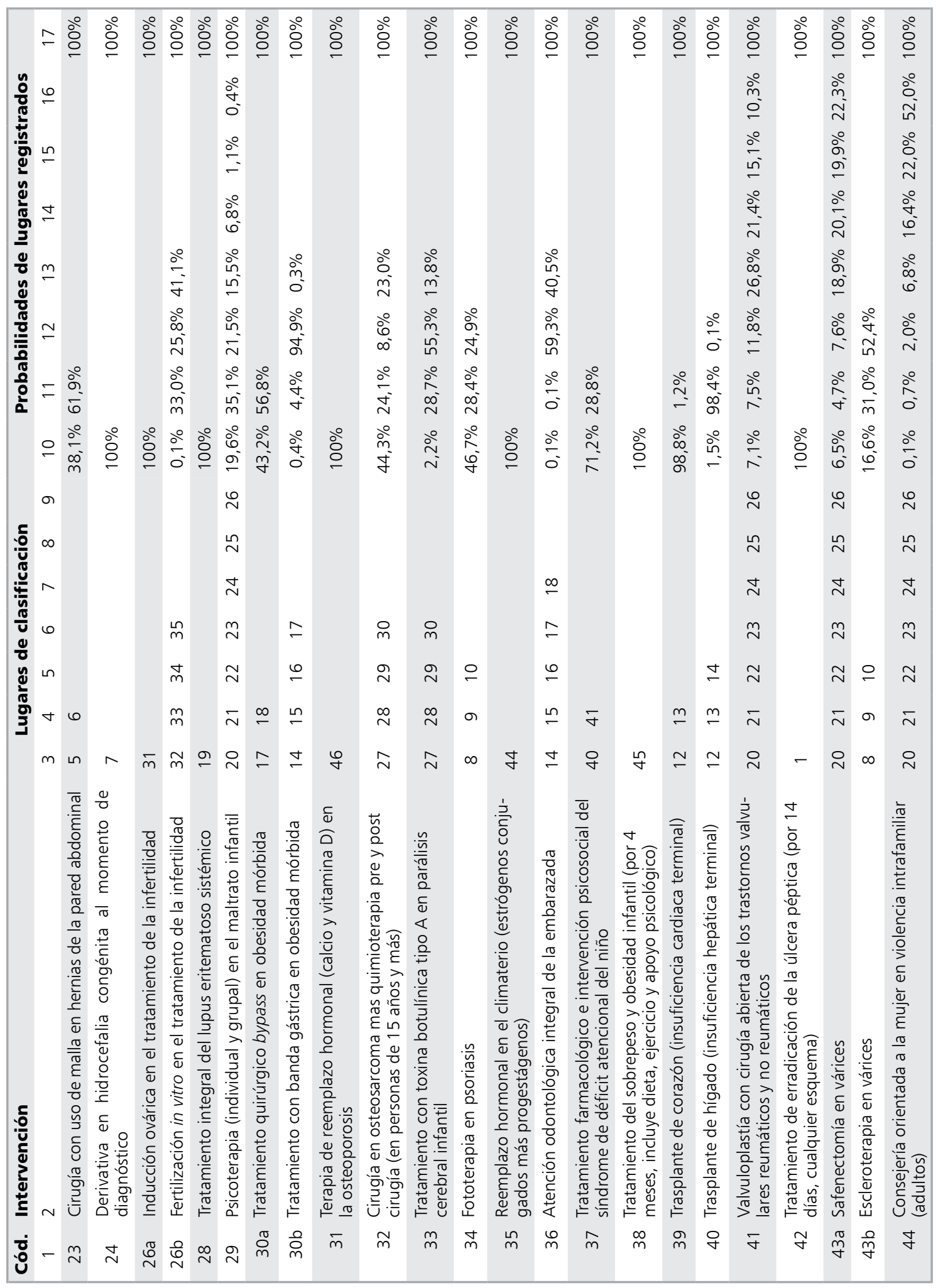




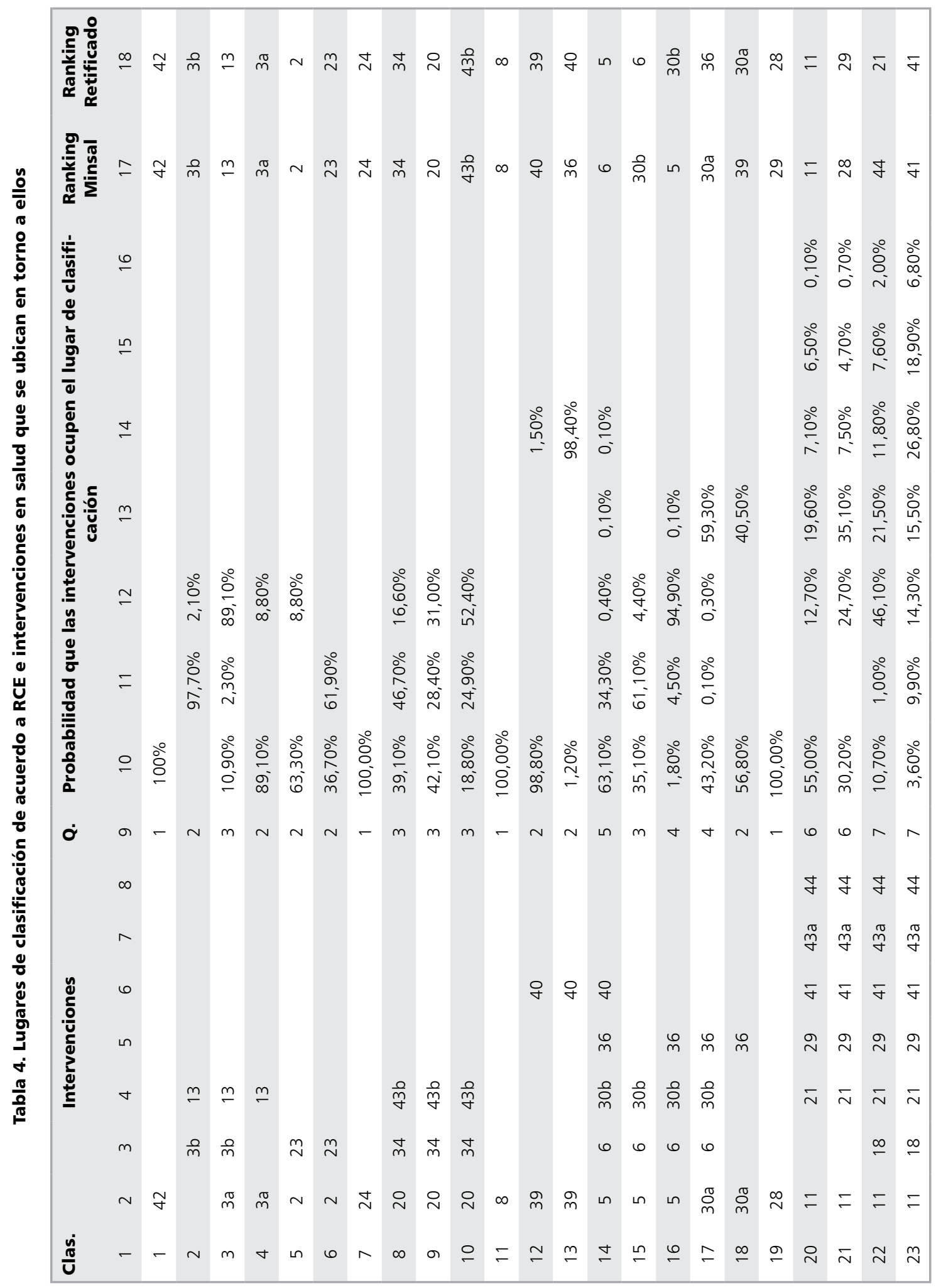




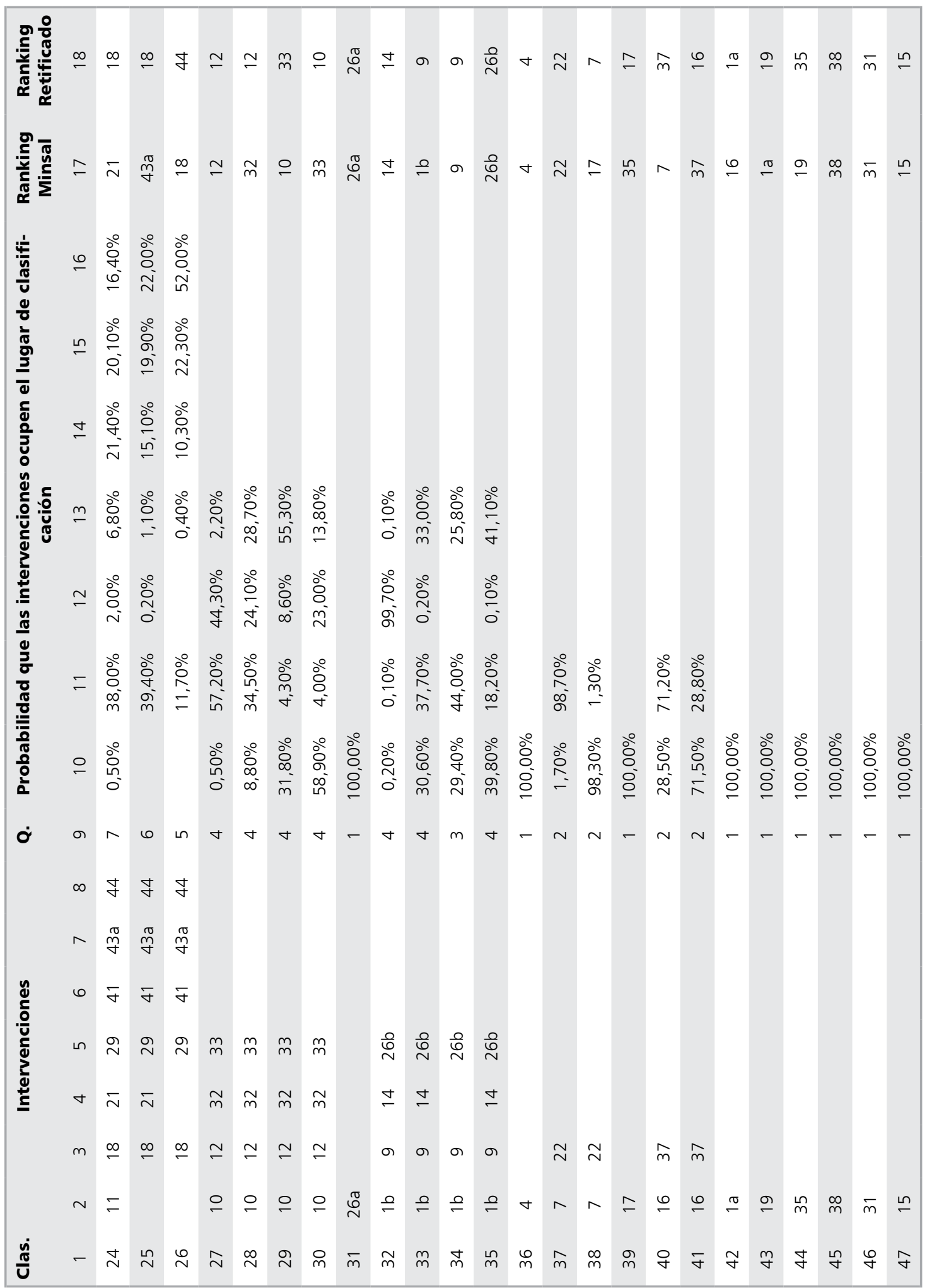


La columna 1 corresponde a los códigos de las intervenciones, la columna 2 registra el nombre de las intervenciones, los posibles lugares de clasificación se muestran desde la columna 3 a la 9. En seguida, desde las columnas 10 a la 16 se muestran las probabilidades de ocurrencia de los respectivos lugares de clasificación indicados anteriormente, $\mathrm{y}$ en el mismo orden. Finalmente, la columna 17 corresponde a la suma de las probabilidades, que debe ser $100 \%$.

Este reporte revela que: i) 13 intervenciones no experimentaron cambio en su clasificación costo-efectividad (figuran con un solo lugar en la clasificación); ii) 10 intervenciones presentan dos posibles lugares de clasificación en el orden costo efectividad; iii) 7 programas presentan tres lugares probables de clasificación; iv) 10 programas tienen probabilidad de ocupar cuatro lugares diferentes; v) 2 intervenciones muestran cinco lugares diferentes de clasificación; vi) 1 programa tiene probabilidad de ocupar seis lugares diferentes y vii) 4 intervenciones tienen probabilidad de ocupar siete lugares diferentes de clasificación.

En general, 34 intervenciones (72\%) presentaron al menos dos lugares posibles de clasificación costo-efectividad.

El segundo reporte diseñado para este estudio, es un reordenamiento del anterior, y muestra los lugares de clasificación y la manera como las diferentes intervenciones sanitarias se agrupan en torno a dichos puestos de orden costo-efectividad.

Este se muestra mediante la Tabla 4. En ella, la primera columna corresponde a los lugares de clasificación del 1 al 47, las columnas dos a la octava muestran las intervenciones que pueden ocupar dichos lugares de clasificación, la columna 9 indica la cantidad de intervenciones que tienen probabilidad de ocupar el lugar indicado de clasificación.

Se puede observar que los lugares 20 al 26 concentran la mayor cantidad de intervenciones. Así, los lugares 22 al 24 de la clasificación, pueden ser ocupados por siete intervenciones; seis programas disputan los lugares 20,21 y 25 y cinco intervenciones compiten por el lugar 26.

Desde la columna 10 a la 16 se muestran las probabilidades de que las intervenciones ocupen los lugares de clasificación indicados, estas aparecen en el mismo orden en que las intervenciones figuran en las columnas anteriores. Esta última información se utilizó para proponer el ranking rectificado de la columna 18 , donde para cada
Tabla 5. Cantidad de lugares de desplazamiento en el orden costo efectividad, cantidad

de intervenciones desplazadas en cada caso y porcentajes

\begin{tabular}{|ccc|}
\hline $\begin{array}{c}\text { Cantidad de } \\
\text { lugares de } \\
\text { desplazamiento }\end{array}$ & $\begin{array}{c}\text { Cantidad de } \\
\text { intervenciones }\end{array}$ & $\%$ \\
\hline 0 & 26 & $55,32 \%$ \\
\hline 1 & 11 & $23,40 \%$ \\
\hline 2 & 6 & $12,77 \%$ \\
\hline 4 & 2 & $4,26 \%$ \\
\hline 5 & 1 & $2,13 \%$ \\
6 & 1 & $2,13 \%$ \\
\hline & 47 & $100,00 \%$ \\
\hline
\end{tabular}

lugar desde el 1 al 47, fue seleccionada aquella intervención que presentó la mayor probabilidad de ocupar el lugar de clasificación.

La columna 17 muestra el ranking publicado por Minsal en 2010, con el propósito de establecer los desplazamientos de lugar de clasificación.

La proposición de ranking rectificado dio como resultado que: 26 intervenciones mantuvieron el mismo lugar en el ranking; 11 intervenciones cambiaron un lugar con respecto al ranking del estudio publicado por el Minsal; 6 intervenciones se desplazaron dos lugares en la clasificación; 2 intervenciones cambiaron 4 lugares. La intervención 35 Reemplazo hormonal en el climaterio cambio desde el lugar 39 al 44 (5 lugares), en tanto que la intervención 39 Trasplante de Corazón se desplazó desde el lugar 18 al 12 (6 lugares).

Como resumen sintético de esta última parte, las cifras de la Tabla 5 muestran que 21,28\% de las intervenciones se desplazaron entre 2 a 6 lugares en el orden de clasificación de costo efectividad, con respecto a la publicado por MINSAL en 2010.

\section{Discusión}

A pesar de que los costos directos continúan representando un porcentaje importante dentro del costo total sanitario de casi todas las intervenciones $^{33}$, este estudio particular, demuestra que las variaciones en CIE tienen el poder de desplazar a las intervenciones dos, cuatro y hasta seis lugares 
en su clasificación según la razón costo efectividad, (tomando como referencia el estudio del Minsal citado).

$\mathrm{Al}$ observar las probabilidades de que las intervenciones ocupen determinados lugares en el orden costo-efectividad, en algunos casos, estas son muy altas para uno o dos lugares y el resto presenta probabilidades más bien bajas de ocurrencia.

Por ejemplo, en el caso del segundo lugar en la clasificación, este puede ser ocupado por las intervenciones $3 \mathrm{~b}$ Cirugía laparoscópica en apendicitis aguda y 13 tratamiento ortopédico de la displasia congénita de cadera. Sin embargo, la intervención con mayores probabilidades de ocupar ese lugar es la $3 \mathrm{~b}(97,7 \%)$ en tanto que la probabilidad de que la intervención 13 ocupe ese lugar es de solamente $2,10 \%$.

Por otro lado, los lugares 8 y 9 presentan probabilidades con menos diferencias entre sí. En particular, el lugar 9 que puede ser ocupado por las intervenciones $20(42,10 \%) ; 34(28,40 \%)$ y $43 \mathrm{~b}(31 \%)$.

Si estuviera tomándose una decisión en la que la intervención 39 Trasplante de corazón (insuficiencia cardiaca terminal), que muestra el lugar 18 en el estudio de Minsal y 12 en el ranking rectificado, seis intervenciones habrían tenido prioridad sobre aquella. Un análisis más exhaustivo en esta intervención que pretende explicar en parte el desplazamiento de 6 lugares muestra en primer lugar que el promedio de tasas utilizadas en el estudio del Minsal, (considerando más de 100 prestaciones incluidas en conjunto en las fases de diagnóstico, tratamiento y seguimiento) fue de $19,5 \%$ en tanto que la tasa más probable de acuerdo a la simulación resulto ser en promedio $11 \%$, tal diferencia provocó una variación en el costo de la intervención de 6,2\% menos, por lo que disminuyó la RCE y de esa forma mejoró su posición. Lo anterior fue acompañado porque las intervenciones circundantes aumentaron sus RCE ocupando lugares más altos en el ranking.

Este tipo de análisis es particularmente importante cuando se trata de analizar la conveniencia de implementar intervenciones versus el escenario nulo. Es decir, en el contexto de estudios de costo-efectividad generalizados, que tienden a resolver cuáles son las intervenciones más efectivas en términos del costo. Las decisiones de incorporación de intervenciones sanitarias al Plan Auge, constituyen un ejemplo ilustrativo en Chile.
Dado los resultados mostrados, hay buenas razones para sostener que las variaciones en costos estructurales tienen un impacto digno de considerar sobre el ranking de costo-efectividad en el conjunto de intervenciones estudiadas. En nuestra opinión, esto va a depender de:

i. Los diferentes perfiles de infraestructura, espacios físicos, servicios, instalaciones, apoyo logístico y administrativo.

ii.Los diferentes criterios de reparto y/o consumo de estos recursos a lo largo y ancho de los centros de responsabilidad de estas organizaciones.

Aun así, surgen preguntas por responder: ¿Qué magnitud de CIE deben tener los hospitales públicos? ¿Qué nivel de consumo energético, servicios, apoyo logístico y administrativo es el más óptimo en relación al servicio que prestan? ¿Cuál es la tasa de CIE más justa y precisa? ¿es posible una estandarización de tasas dependiendo de tipos de hospitales? Estas interrogantes sugieren la necesidad de mayor investigación en torno a este tema lo que en definitiva redundará en mejores evaluaciones económicas de intervenciones sanitarias.

\section{Referencias}

1. Drummond MF, Sculpher MJ, Claxton K, Stoddart GL, Torrance GW. Methods for the Economic Evaluation of Health Care Programmes. Furth Edition. Oxford: Oxford University Press, 2015. Pag. 1-166.

2. Sculpher MJ, Price M. Measuring costs and consequences in economic evaluation in asthma. Respir Med 2003 May; 97 (5): 508-20.

3. Zárate V. Economic evaluations in healthcare: Basic concepts and classification. Rev Med Chile 2010; 138: 93-7.

4. Saldivia S, Vicente B, Torres F. Measuring outcomes in economic evaluations. Rev Med Chile 2010; 138: 79-82.

5. Castillo-Laborde C. Economic evaluation in the health care decision making process: The case of England. Rev Med Chile 2010; 138: 103-7.

6. Castillo-Riquelme M, Zárate V. Economic evaluation in Healthcare. Rev Med Chile 2010; 138: 67-8.

7. Sculpher MJ, Price M. Measuring costs and consequences in economic evaluation in asthma. Respiratory Medicine 2003; 97: 508-20.

8. Vallejos C, Bustos L, De La Puente C, Reveco R, Velásquez M, Zaror C. (2014). "Principales aspectos metodológicos en la Evaluación de Tecnologías Sanitarias”. Rev Med Chile 142 (S1): S 16-21. 
9. Olsen JA. Principles in Health Economics and Policy. Oxford, UK: Oxford University Press; 2009. Pag. 175205.

10. Alvis N, Valenzuela MT. QALYs and DALYs as synthetic indicators of health. Rev Med Chile 2010; 138: 83-7.

11. Rushby JF, Hanson K. Calculating and presenting disability adjusted life years (DALYs) in cost-effectiveness analysis. Health Policy and Planning 2001; 16: 326-31.

12. Eichler HG, Kong SX, Gerth WC, Mavros P, Jonsson B. Use of cost-effectiveness analysis in health-care resource allocation decision-making: How are cost-effectiveness thresholds expected to emerge? Value in Health 2004; 7: 518-28.

13. Vander Plaetse B, Hlatiwayo G, Van Eygen L, Meessen $\mathrm{B}$, Criel B. Costs and revenue of health care in a rural Zimbabwean district. Health Policy and Planning 2005; 20: 243-51.

14. Tan SS, van Ineveld BM, Redekop WK, Hakkaart-van Roijen L. Comparing Methodologies for the Allocation of Overhead and Capital Costs to Hospital Services. Value in Health 2009; 12: 530-5.

15. Carreras M, García-Goni M, Ibern P, Coderch J, Va11-Llosera L, Inoriza JM. Estimates of patient costs related with population morbidity: can indirect costs affect the results? European Journal of Health Economics 2011; 12: 289-95.

16. Angelis A, Kanavos P, López-Bastida J, Linertova R, Nicod E, Serrano-Aguilar P, et al. Social and economic costs and health-related quality of life in non-institutionalised patients with cystic fibrosis in the United Kingdom. Bmc Health Services Research 2015; 15.

17. Atif M, Sulaiman SAS, Shafie AA, Asif M, Babar ZUD. Resource utilization pattern and cost of tuberculosis treatment from the provider and patient perspectives in the state of Penang, Malaysia. Bmc Health Services Research 2014;14.

18. Baboolal K, McEwan P, Sondhi S, Spiewanowski P, Wechowski J, Wilson $\mathrm{K}$. The cost of renal dialysis in a UK setting - a multicentre study. Nephrology Dialysis Transplantation 2008; 23: 1982-9.

19. Bayati M, Ahari AM, Badakhshan A, Gholipour M, Joulaei H. Cost Analysis of MRI Services in Iran: An Application of Activity Based Costing Technique. Iranian Journal of Radiology 2015; 12.

20. Chen A, Sabharwal S, Akhtar K, Makaram N, Gupte CM. Time-driven activity based costing of total knee replacement surgery at a London teaching hospital. Knee 2015; 22: 640-5.

21. Dalaba MA, Akweongo P, Savadogo G, Saronga H, Williams J, Sauerborn R, et al. Cost of maternal health services in selected primary care centres in Ghana: a step down allocation approach. Bmc Health Services Research 2013; 13.

22. Elamin EI, Ibrahim MIM, Sulaiman SAS, Muttalif AR. Cost of illness of tuberculosis in Penang, Malaysia. Pharmacy World \& Science 2008; 30: 281-6.

23. Ezenduka $\mathrm{C}$, Ichoku $\mathrm{H}$, Ochonma O. Estimating the Costs of Psychiatric Hospital Services at a Public Health Facility in Nigeria. Journal of Mental Health Policy and Economics 2012; 15: 139-48.

24. Henry SG, Ness RM, Stiles RA, Shintani AK, Dittus RS. A cost analysis of colonoscopy using microcosting and time-and-motion techniques. Journal of General Internal Medicine 2007; 22: 1415-21.

25. Hu FC, Wang JD. The development of cost-effectiveness indices with equity implications for the economic evaluation of health care. Value in Health 2008; 11: A172-A.

26. Jiménez DJ, Bastías SG. The scope of economic evaluation of healthcare interventions. Rev Med Chile 2010; 138: 71-5.

27. Khan A, Zaman S. Costs of vaginal delivery and Caesarean section at a tertiary level public hospital in Islamabad, Pakistan. Bmc Pregnancy and Childbirth 2010; 10.

28. Miller TL, McNabb SJN, Hilsenrath P, Pasipanodya J, Drewyer G, Weis SE. The Societal Cost of Tuberculosis: Tarrant County, Texas, 2002. Annals of Epidemiology 2010; 20: 1-7.

29. Negrini D, Sheppard L, Mills GH, Jacobs P, Rapoport J, Bourne RS, et al. International Programme for Resource Use in Critical Care (IPOC) - a methodology and initial results of cost and provision in four European countries. Acta Anaesthesiologica Scandinavica 2006; 50: 72-9.

30. Roberts RR, Kampe LM, Hammerman M, Scott RD, Soto T, Ciavarella GG, et al. The cost of care for patients with HIV from the provider economic perspective. Aids Patient Care and Stds 2006; 20: 876-86.

31. Shander A, Hofmann A, Ozawa S, Theusinger OM, Gombotz H, Spahn DR, et al. Activity-based costs of blood transfusions in surgical patients at four hospitals. Transfusion 2010; 50: 753-65.

32. Sharara N, Adam V, Crott R, Barkun AN. The costs of colonoscopy in a Canadian hospital using a microcosting approach. Canadian Journal of Gastroenterology 2008; 22: 565-70.

33. Van Minh H, Giang KB, Huong DL, Huong LT, Huong NT, Giang PN, et al. Costing of clinical services in rural district hospitals in northern Vietnam. International Journal of Health Planning and Management 2010; 25: 63-73.

34. Vertuani S, Nilsson J, Borgman B, Buseghin G, Leonard C, Assietti R, et al. A Cost-Effectiveness Analysis of Mi- 
nimally Invasive versus Open Surgery Techniques for Lumbar Spinal Fusion in Italy and the United Kingdom. Value in Health 2015; 18: 810-6.

35. Ministerio de Salud de Chile. Estudio de Costo Efecti- vidad de Intervenciones en Salud. Informe Final 2010. Disponible en http://desal.minsal.cl/publicaciones/ evaluacion-economica-de-intervenciones-en-salud/ [consultado el 17 de octubre de 2016]. 